\title{
Propuesta metodológica para evaluar procesos de trabajo en los Departamentos de Vigilancia y Lucha Antivectorial
}

\author{
Methodological proposal for the evaluation of the working \\ processes in the anti-vector surveillance and fight departments
}

\section{Dra. Vivian de las Mercedes Noriega Bravo}

Escuela Nacional de Salud Pública. La Habana, Cuba.

\section{RESUMEN}

La evaluación de los Departamentos de Vigilancia y Lucha Antivectorial es una necesidad que está dada en el encargo que estos tienen en las áreas de salud y los problemas detectados en su desempeño. Las escasas evidencias científicas sobre la labores que realizan, muestran que los esfuerzos se han dirigido mayormente a evaluar aspectos específicos de sus programas, como es la idoneidad de las actividades, la adecuación de los recursos y los resultados en relación con determinados objetivos, pero no, hacia cómo se gestionan los procesos. Sin embargo, los frecuentes eventos epidemiológicos debido al incremento en los índices de infestación por el Aedes aegyti suscita la necesidad de una mirada holística sobre los procesos de trabajo que se desarrollan para controlar el vector. El objetivo de este artículo es mostrar los pasos seguidos en el diseño de una propuesta metodológica para evaluar los departamentos de Vigilancia y Lucha Antivectorial desde los procesos que realizan para el control del Aedes aegypti en el nivel primario de atención de salud. Esta propuesta tiene sus fundamentos en evaluaciones diagnósticas y está diseñada con la suficiente flexibilidad para ser adaptada y utilizada en otras unidades organizativas del área de Higiene y Epidemiología. Se espera que la aplicación de esta metodología permita determinar aspectos organizativos y funcionales de los Departamentos de Vigilancia y Lucha Antivectorial, que pudieran estar incidiendo en la calidad de su trabajo y en consecuencia, apoyaría la toma de decisiones certeras.

Palabras clave: evaluación, procesos, vigilancia y lucha antivectorial, Aedes aegypti, control vectorial. 


\section{ABSTRACT}

The evaluation of the anti-vector surveillance and fight departments is a necessity resulting from their tasks in the health areas and the problems detected in their performance. The little scientific evidence on the work of these departments show that the efforts have been so far directed to evaluate specific aspects of their programs such as suitability of activities, adequacy of resources and results according to particular objectives; but not to how the working processes are managed. However, the frequency of epidemiological events due to the increased infestation indexes by Aedes aegypti mosquitoes makes it necessary to take a holistic look at the working processes to control the vector. The objective of this article was to show the steps followed in the design of a methodological proposal for the evaluation of the anti-vector surveillance and fight departments on the basis of the control processes of Aedes aegypti at the primary health care. The methodological proposal was substantiated on diagnostic evaluations and was designed to be flexible enough to adapt and use it in other organizational units of the hygiene and epidemiology sphere. It is expected that the implementation of this methodology allow determining the organizational and functional elements of the above-mentioned departments, which could have an effect on the quality of work and the effectiveness of the decisionmaking.

Key words: evaluation, processes, anti-vector surveillance and fight, Aedes aegypti, vector control.

\section{NTRODUCCI ÓN}

Los Departamentos de Vigilancia y Lucha Antivectorial (DVLA) integran los servicios de Higiene y Epidemiología de las áreas de salud ${ }^{1}$ y obviamente son parte de los sistemas municipales de salud, por lo que sus actividades conforman también el conjunto de procesos que contribuyen al cumplimiento de la misión de estos; son unidades organizativas estratégicas a ese nivel, porque su accionar está dirigido a la vigilancia y control de vectores biológicos que tienen marcada importancia en el comportamiento del cuadro de salud.

Tienen un encargo que cumplir en el nivel primario de atención de salud y para ello realizan múltiples actividades y tareas derivadas de diferentes programas nacionales, entre ellos, el más importante de todos es el Programa Nacional de Erradicación del Aedes aegypti (PNEAa).

Como en toda unidad organizativa, Ios DVLA desarrollan procesos a partir de actividades en los que intervienen personas, materiales, equipos, técnicas y procedimientos, para producir resultados de trabajo; son por tanto, agrupaciones humanas constituidas para alcanzar objetivos específicos, con determinado nivel de especialización de sus miembros, los que desarrollan actividades aplicando recursos en pos de ciertos valores comunes en un marco de trabajo normado administrativa, metodológica y jurídicamente y que responden a una estructura dentro de un contexto al que influencia parcialmente. 
En el quehacer de los DVLA se han constatado dificultades en cuanto a la calidad de las labores que realizan y la permanencia de su personal, entre otros, ${ }^{2,3}$ y en respuesta a estas situaciones, se han desarrollado acciones encaminadas a solventar dificultades, tales como capacitaciones, mejoras en las condiciones de trabajo, aumentos salariales, cambios estructurales y otros.

Aun con los elementos que hacen cuestionable el desempeño de los DVLA en términos de gestión y calidad, son escasos los estudios orientados a valorar las actividades que estos realizan de manera integral, en el cúmulo de procesos que se producen o deben producirse a nivel del área de salud. De ahí que el objetivo de este trabajo es mostrar los principales fundamentos teóricos y metodológicos y los pasos seguidos en el diseño de una propuesta metodológica para evaluar los referidos departamentos en cuanto a los procesos que realizan para el control del Aedes aegypti.

\section{FUNDAMENTOS DE LA PROPUESTA METODOLÓGI CA PARA EVALUAR LOS DEPARTAMENTOS DE VI GI LANCI A Y LUCHA ANTI VECTORI AL}

En toda organización se dan interacciones entre los elementos que conforman el sistema de trabajo, entre todos los grupos de persona que integran sus unidades organizativas, guiadas por mecanismos que exceden fronteras funcionales y estructurales; por tanto, el éxito del policlínico depende cada vez más de la atención que se de a la interrelación de los procesos que se desarrollan en él. La identificación y análisis de manera integral de los procesos a fin de ser perfeccionados en términos de calidad garantiza una proyección y un desempeño más eficaz y más eficiente. ${ }^{4}$

En el presente trabajo se asume la definición más general de procesos, la cual responde a la repercusión que tienen en la calidad, entre ellos están: ${ }^{5,6}$

- Procesos fundamentales o clave, son las actividades esenciales que identifican y distinguen a la entidad, los relacionados directamente con la misión y justifican la existencia del servicio, "su razón de ser."

- Procesos de soporte o apoyo, son los encargados de proveer a la organización de los medios necesarios (recursos materiales, humanos y financieros) y crear las condiciones para que el proceso clave tenga lugar de forma efectiva. Son actividades secundarias de la cadena de valor que pueden ser determinantes para alcanzar los objetivos propuestos.

- Procesos estratégicos, son los que proporcionan directrices y límites de actuación al resto de los procesos, generalmente realizados por la dirección y permiten definir, mantener y desplegar las políticas y estrategias de la organización.

Los procesos para el control del Aedes aegypti son entendidos como un conjunto de múltiples interacciones y coordinaciones humanas y materiales que se desarrollan entorno al PNEAa en el nivel primario de atención de salud, a fin de conseguir una situación "mejor" con respecto al nivel de infestación por el vector hasta lograr su total eliminación.

En la práctica, el desempeño de los DVLA se materializa en la visita periódica a viviendas, escuelas, centros de trabajo y otros locales en busca de focos potenciales y reales del Aedes aegypti, la aplicación de diferentes formas de tratamiento físico, químico o biológico para la prevención y destrucción de criaderos, así como la eliminación de las formas adultas del vector mediante tratamientos especiales. Esto 
lleva consigo, medidas que regulan el cumplimiento de la legislación sanitaria vigente y permiten sancionar a las personas que con actitudes negligentes las infrinjan.

De la actuación de los operarios integrales de control de vectores se genera información, la que se gestiona a partir de mecanismos que permiten su difusión para ser utilizada en el proceso de toma de decisiones y en la implementación de medidas correctoras administrativas y legales, con lo que se define un componente organizacional importante para el accionar en el área de salud.

La combinación armónica de estos procederes técnicos y de los mecanismos de gestión que agregan valor interno, y exceden las fronteras funcionales del DVLA, define uno de los procesos principales que incide en el cumplimiento del encargo que tienen las áreas de salud; y que en este trabajo se suscribe al proceso de control vectorial en fase preadulta, que se define como:

El conjunto de actividades interrelacionadas, técnicas y gerenciales, que desarrollan los Departamentos de Vigilancia y Lucha Antivectorial a fin de prevenir y detectar oportunamente la presencia de focos de Aedes aegypti en la totalidad de los locales que conforman su universo de trabajo en el área de salud.

Por el carácter preventivo de las actividades que conforman este proceso, es vital que las mismas se integren en la gestión del conjunto de procesos que se desarrollan a nivel del área de salud, mediante el establecimiento de mecanismos que guíen las interacciones y el manejo sistemático de la información. La persistencia de factores ambientales, sociales, comportamentales, organizacionales y otros, que dificultan en Ios DVLA la realización de actividades a tal efecto, demanda que las mismas se gestionen y valoren como procesos y no de forma aislada, lo cual ha prevalecido hasta el momento.

\section{SEÑO DE LA PROPUESTA METODOLÓGI CA PARA EVALUAR LOS DEPARTAMENTOS DE VI GI LANCI A Y LUCHA ANTI VECTORI AL DESDE SUS PROCESOS DE TRABAJO}

En la actualidad, una de las maneras de concebir la evaluación de organizaciones es como una investigación de tipo valorativo sujeta a las premisas del método científico propio de las ciencias sociales, ${ }^{7}$ y desde una perspectiva amplia se le concede a la misma el alcance para emitir juicios de valor en cuanto desempeño en la realización de los procesos de trabajo a efectos de contrastar elementos favorecedores o que obstaculizan, y en consecuencia, proporcionar aquellos que apoyen a la corrección o no de desviaciones y el fortalecimiento de los logros.

Una de las situaciones enfrentadas ante la interrogante de ¿cómo evaluar los procesos en los DVLA? fue la no disponibilidad de un sistema de evaluación a tal fin. Las evaluaciones organizacionales llamadas "de diagnóstico". ${ }^{8}$ permiten un análisis de los DVLA desde la perspectiva sistémica, de manera que no se evalúan actividades aisladas, sino dentro del conjunto de procesos que realizan en su escenario de actuación; lo que puede ejecutarse a un nivel parcial, en el que se delimita la evaluación a un aspecto específico de los departamentos como puede ser la gestión de los procesos. De ahí que la presente propuesta metodológica tenga sus fundamentos en evaluaciones diagnósticas.

Los pasos de la propuesta se explican a continuación. 


\section{Primer paso: caracterización de los DVLA}

Obtener un conocimiento elemental del objeto de estudio es imprescindible y el primer paso de la propuesta, la cantidad y tipos de variables de estudio empleadas en esta caracterización estarán determinados por los objetivos de la investigación e intereses de los investigadores. En este diseño se obtuvo información sobre las funciones, actividades, normas generales y modalidades de trabajo, técnicas y procedimientos específicos establecidos en el programa de trabajo de los DVLA, así como las obligaciones en cuanto al PNEAa, desde diversas fuentes y con el uso de diferentes técnicas, tales como:

- Normativas y documentos legales, entre los que estuvieron el mencionado PNEAa, ${ }^{9}$ el Programa de Sostenibilidad para la Prevención del dengue y Erradicación del Aedes aegypti, ${ }^{10}$ el Manual para responsables de brigadas y visitadores, ${ }^{11}$ Metodología de encuestas ${ }^{12}$ y Técnica de lucha anti aegypti, ${ }^{13}$ el documento titulado "Integración de la Higiene y la Epidemiología a la Atención Primaria de Salud," 14 y el Manual de organización de policlínico. ${ }^{15}$

- La Resolución 135 del Ministro de Salud Pública sobre Reglamento General de Policlínico, ${ }^{2}$ y otros.

- Estudio del desempeño de los miembros de un DVLA seleccionado de la provincia La Habana en cuanto al cumplimiento de las normas de trabajo a partir del método de observación participante. ${ }^{16}$

- Dinámica grupal con expertos en vigilancia y lucha antivectorial para la identificación de problemas de funcionamiento concernientes a la idoneidad de la actividad de dirección y control de los departamentos, la estimulación y motivación del personal, el aseguramiento de los recursos materiales, el apoyo y reconocimiento social a la labor que realizan, las afectaciones del clima organizacional y la elaboración y análisis de Matriz de Balance de Campo de Fuerzas Innovada. ${ }^{16}$

- Análisis causal de los problemas vinculados al desempeño de los DVLA mediante la técnica de Ishikawa. Se identificaron como factores principales contribuyentes al deficiente desempeño, la inestabilidad de la fuerza laboral, las incorrecciones en la calidad del trabajo que realizan los operarios/as, así como en la conducción y control de estas acciones, y el insuficiente apoyo al trabajo antivectorial. En cada uno de estos, tomados como base los problemas que hasta entonces habían sido detectados, se identificaron aquellos más importantes considerados como causas.

\section{Segundo paso: definición de procesos a evaluar}

La no disponibilidad de un "mapeo" o lista general de los procesos que se realizan en los DVLA y que pudiera ser tomada como referencia para la evaluación, así como la definición poco clara de qué procesos interesa mejorar y cuáles son las potencialidades de perfeccionamiento, limitaron su selección en el diseño. En tal sentido, acorde a lo planteado en la literatura se tomó como base el inventario de problemas existentes, además, la relación de actividades principales identificadas en el paso anterior toda vez que el proceso clave es indispensable para evaluar los DVLA y este lo conforman las actividades principales que inciden en el cumplimiento de su encargo.

Cabe señalar que en un primer momento, se hicieron algunas consideraciones que guiaron el empeño, entre ellas, que en los DVLA existe un proceso principal que identifica el conjunto de actividades básicas que constituye la esencia misma del 
objeto de estudio, su "razón de ser" en el área de salud y por tanto, se justifica que la evaluación se centre en él; y que dada la situación existente, concurren otros procesos involucrados en apoyar o crear las condiciones que hacen viable el proceso principal.

El proceder para la selección de los procesos a evaluar se realizó en consulta con expertos mediante el método Delphi, el que se escogió por su utilidad en aplicaciones de consenso a partir del desarrollo de un proceso de convergencia de opiniones, mediante rondas sucesivas de entrevistas anónimas y con la máxima autonomía por parte de los participantes. Es útil porque, entre otros elementos, permite la participación de personas ubicadas en lugares alejados y brinda un mayor tiempo a los expertos consultados para elaborar sus respuestas. ${ }^{17,18}$

Para el desarrollo de la técnica Delphi se atendió a lo recomendado por la literatura y se conformó un panel con 18 jueces expertos, personas capaces de ofrecer valoraciones conclusivas del asunto tratado y hacer recomendaciones con un máximo de competencias; por lo cual se tuvieron como requisitos básicos para su selección, poseer el nivel de conocimiento requerido y experiencia práctica en el trabajo de vigilancia y lucha antivectorial, entre ellos estuvieron especialistas de Higiene y Epidemiología, Medicina General Integral y Administración y Organización de Salud, biólogos, tecnólogos de la salud y técnicos en Higiene y Epidemiología, todos avalados por su buena preparación técnica y permanencia por diez o más años vinculados a la actividad.

Acorde a las exigencias de la técnica, se solicitó la colaboración de todos a través de mensaje electrónico y se les brindó la información requerida en cuanto a los objetivos del estudio y la metodología a seguir. Se cumplió con el anonimato entre los participantes y se logró el consenso en tres rondas de entrevistas; se consideró como criterio de aceptación y poder pasar de una a otra ronda, el 80 \% o más de consenso.

La técnica se aplicó en dos momentos de la investigación; primero un grupo de 11 expertos validó el grupo de actividades ya referidas, las cuales resultaron ser:

1. Conducir el pase de revista.

2. Inspeccionar los locales ubicados en el área de trabajo.

3. Ejecutar los tratamientos correspondientes a los depósitos.

4. Realizar vigilancia en las zonas de riesgo.

5. Instruir a las personas en la realización del autofocal.

6. Atender al completamiento de la fuerza laboral.

7. Atender la preparación técnica del personal.

8. Ajustar el trabajo diario de las brigadas.

9. Controlar el trabajo de las brigadas en el terreno.

10. Asegurar la disponibilidad de los recursos materiales.

11. Comprobar la calidad de las acciones realizadas por los operarios/as. 
12. Velar el cumplimiento del plan de verificación y tratamiento de locales.

13. Analizar el cumplimiento de los indicadores del programa.

14. Analizar los indicadores de productividad de las brigadas y sus operarios.

15. Actualizar la información establecida.

16. Conciliar con el nivel superior las orientaciones del ciclo.

17. Conciliar con el vicedirector la higiene ambiental y los viajeros que arriban.

18. Participar en el Puesto de Mando.

19. Atender a las necesidades de alimentación, vestuario y otras condiciones de trabajo.

20. Conciliar con los factores de la comunidad el apoyo a las tareas.

21. Intercambiar información de las inspecciones con el médico de familia.

En otro momento, los restantes siete expertos valoraron y aprobaron la propuesta de procesos clave, de soporte y estratégicos, realizada por un grupo de especialistas de Epidemiología con los cuales se realizó previamente una dinámica grupal para analizar cada una de las actividades antes mencionadas, y en base a los requisitos establecidos para considerarse un proceso, proponer aquellos que pudieran ser evaluados en los DVLA.

Como resultante de la consulta con los expertos se definió como procesos a evaluar en la aplicación de la propuesta (recuadro) lo siguiente:

Recuadro. Procesos a evaluar

\begin{tabular}{|l|l|}
\hline Procesos clave & $\begin{array}{l}\text { Proceso de control vectorial en fase preadulta y diferenciado, el } \\
\text { conjunto de actividades organizativas y de control que ejecutan } \\
\text { los miembros de la cadena de mando como el subproceso de } \\
\text { organización y ejecución del mando. }\end{array}$ \\
\hline $\begin{array}{l}\text { Procesos de } \\
\text { soporte o apoyo }\end{array}$ & $\begin{array}{l}\text { Aseguramiento material. } \\
\text { Interrelación con unidades organizativas de salud. } \\
\text { Interrelación con instituciones representativas de otros sectores } \\
\text { de la sociedad y la economía. }\end{array}$ \\
\hline \begin{tabular}{l|l} 
Procesos \\
estratégicos
\end{tabular} & $\begin{array}{l}\text { Gestión de los recursos humanos. } \\
\text { Gestión del ambiente laboral. }\end{array}$ \\
\hline
\end{tabular}

\section{Tercer paso: definición de criterios para valorar los procesos propuestos}

Los procesos pueden ser medidos en virtud de las condiciones de las que se parte, el grado en que estos se ajustan a los requisitos establecidos y los resultados que se consiguen $\mathrm{y}$, desde esta perspectiva, se tiene diversidad de criterios, los que no son más que las características observables que conciernen a lo que se evalúa. ${ }^{19}$

http://scielo.sld.cu 
Su elección, al igual que el estándar, el cual limita lo que se acepta o no respecto a este, debe ser rigurosa puesto que tiene repercusión en la interpretación del grado de cumplimiento del proceso que se va a estudiar y por ende, en las personas que lo realizan. Establecer un estándar significa suponer un nivel de calidad cuyo grado de cumplimiento es medido, en forma cuantitativa o cualitativa, a partir de indicadores o el comportamiento regular de dimensiones en los criterios evaluados.

A tal efecto, se trabajó en la elaboración de los criterios evaluativos para cada uno de los procesos que se propusieron. Todas las actividades se agruparon a partir de que los criterios englobaran tanto el componente técnico como gerencial que conforman el proceso clave de control vectorial en fase preadulta, y fueran capaces de aportar información para medir todas las actividades referentes a su desarrollo, lo que incluye las actividades concernientes a los procesos estratégicos y de soporte que los apoyan; y con ello, dar juicios de valor sobre el desempeño de los DVLA en las áreas de salud.

Tanto en los criterios como la forma en que estos serían medidos, se tomó en cuenta la coherencia con el marco teórico y los objetivos de investigación, la disponibilidad de fuentes para proveer los datos necesarios y la sencillez de su procesamiento. Se procuraron criterios inteligibles, razonablemente aceptados por las personas a quienes se dirigen los resultados y que contribuyeran a la toma de decisiones.

El conocimiento sobre los factores ambientales, sociales, comportamentales, organizacionales y otros, que dificultan la realización de las actividades en los DVLA, acumulado desde la práctica y los estudios realizados durante los años de ejecución del proyecto de ISSS (del cual es resultante esta investigación), sirvió de base para la consecución de estos atributos.

Este esfuerzo contó con la contribución de profesores de la Escuela Nacional de Salud Pública, autorizados especialistas en el campo de la evaluación epidemiológica de los servicios de salud y la de tipo organizacional, con los que se sostuvieron varios encuentros en los que se discutió y llegó a acuerdos que permitieron realizar ajustes sucesivos a la relación de criterios en la medida de su construcción.

Por último, antes de la versión final, los criterios propuestos se sometieron a la consideración de siete expertos en el área de trabajo de la vigilancia y lucha antivectorial, personas reconocidas por sus conocimientos teóricos y la práctica como directivo o miembros de la cadena de mando en los referidos servicios, por más de diez años.

\section{Cuarto paso: identificación de técnicas e instrumentos para recopilar información}

La recolección de información necesaria constituye la base de cualquier evaluación, por tanto, la selección y aplicación de las técnicas apropiadas debe hacerse de manera cuidadosa para que entre otras cosas, se garantice la validez de las conclusiones a las que se arribe.

En virtud de que uno de los referentes teóricos para el diseño de la propuesta metodológica fue el estudio de caso, se consideró conveniente la combinación de técnicas cuantitativas y cualitativas a fin de hacer más comprensible el desempeño del DVLA que se estudie, desde la realización de sus procesos de trabajo. Entre las técnicas empleadas y propuestas para la evaluación están:

- Revisión documental, para el estudio de la plantilla de personal y obtener información acerca de las plazas ocupadas y disponibles según categorías, lo que 
unido a la revisión de los expedientes laborales, los partes diarios de actividades, informes de cierre de ciclos de trabajo y otros documentos, permite acopiar datos relacionados con la gestión de los recursos humanos y de materiales.

- Observación participante, ajena, no encubierta y semiestructurada, es la modalidad que se utiliza a fin de explorar las actividades inherentes al proceso de control vectorial en fase preadulta. Para su desarrollo se requiere de personas con un grado de entrenamiento en las actividades que se van a observar y el uso de una guía que apoye las observaciones. A tal efecto se creó y validó una guía de observación que tuvo sus bases en documentos normativos y argumentos emitidos por personas competentes en esta área de trabajo, sobre las actividades que debían observarse durante la actuación de operarios/as, miembros de la cadena de mando y el vicedirector de Higiene y Epidemiología en el desarrollo de los procesos de control vectorial en fase preadulta. La misma se acompaña de un instructivo en el que se detallan técnicas y procederes que hacen uniformes y precisos los juicios del observador. ${ }^{20}$

- Cuestionario, utilizado para proveer los datos sobre la gestión el ambiente laboral.

El ambiente laboral o de trabajo está dado en el conjunto de factores, circunstancias, las condiciones del medio bajo las cuales las personas desarrollan su actividad laboral, los que pueden ejercer influencia positiva y negativa, según sean percibidos por los trabajadores, y producir un determinado clima. ${ }^{21,22}$

El hecho que la inestabilidad del personal sea uno de los problemas más comunes en los DVLA y que la cierta "autonomía" con que realizan las labores requiera un ambiente de confianza y compromiso, plantea que el ambiente laboral en estos deba ser gestionado como un recurso más. Por esas razones en la propuesta metodológica se evalúa el proceso de gestión del ambiente laboral a través de variables que expresan el clima organizacional existente, entendido este como la percepción que tienen los miembros de los DVLA sobre los distintos elementos de relativa permanencia que conforman el ambiente en el cual realizan su trabajo, en términos de la estructura y los procesos organizacionales como liderazgo, toma de decisiones y motivacionales.

Durante la caracterización de los DVLA se construyó y validó el instrumento que satisface tal necesidad. ${ }^{23}$

- Entrevista no estructurada y semiestructurada resultan factibles de aplicar en este ámbito de trabajo. Con ellas pueden establecerse diálogos "profundos" con los directivos del área de salud, miembros de la cadena de mando, representantes de entidades, organismos y organizaciones de masas en el territorio que por sus conocimientos y la posición que ocupan sean considerados informantes clave y recoger así información acerca de los diferentes procesos desde sus testimonios.

\section{COMENTARI O FI NAL}

La propuesta metodológica que se presenta para evaluar los DVLA desde los procesos que realizan en el control del Aedes aegypti a nivel de las áreas de salud tiene sus bases en evidencias prácticas y elementos probados científicamente. 


\section{REFERENCI AS BI BLI OGRÁFICAS}

1. Ministerio de Salud Pública. Resolución No. 135. Reglamento General del policlínico. La Habana; 17 de junio de 2008. Disponible en: http://files.sld.cu/minsapdocumentos/2009/08/13/resolucion-ministerial-no-135\% $2 \% 80 \%$ 93-reglamento-general-de-policlinico/

2. Noriega V, Ramos I, Couterejuzón L, Martín L, Mirabal M, Díaz G. Situación organizacional de los Grupos de Control de Vectores en Ciudad de La Habana. Rev Cubana Salud Pública [serie en Internet]. 2009 [citado 25 nov 2009]; 35(2). Disponible en: http://scielo.sld.cu/scielo.php?script=sci_arttext\&pid=S086434662009000200018\&lng=es

3. Noriega V, Ramos I, Morejón Y, Molina R. Evaluación del tratamiento focal contra el Aedes aegypti en áreas de salud seleccionadas de Ciudad de La Habana, junio de 2008. Rev Cubana Hig Epidemiol [Internet] 2009 [citado 25 Nov 2010]; 47(2). Disponible en: http://scielo.sld.cu/scielo. php?script=sci_arttext\&pid=S1561$30032009000200002 \& \operatorname{lng}=\mathrm{es}$

4. Mallar Miguel Á. La gestión por procesos: un enfoque de gestión eficiente. Vis Futur [Internet]. 2010 [citado10 J un 2011]; 13(1). Disponible en:

http://www.scielo.org.ar/scielo.php?script=sci arttext\&pid=S1668$87082010000100004 \&$ lng $=e s \& n r m=i s o \& t \mid n g=e s$

5. Gil Y, Vallejo E. Técnicas de Calidad y Planificación Estratégica. Guía para la identificación y análisis de los Procesos de la Universidad de Málaga. [Internet]. 2008 [citado 6 Feb 2010]. Disponible en: http://www.uma.es/publicadores/gerencia_a/wwwuma/guiaprocesos1.pdf

6. Cuenca LI. Enfoque de procesos. Modelo de referencia [Internet]. [citado 31 May 2010]. Disponible en: http://www.upv.es/ jmontesa/sio/SIO-04-3-txt.pdf

7. Cano Ramírez A. Elementos para una definición de evaluación [Internet]. 2005 [citado 4 Feb 2012]. Disponible en:

http://www.ulpgc.es/hege/almacen/download/38/38196/tema_5_elementos_para_un a_definicion_de_evaluacion.pdf

8. Trinidad Requena A. La evaluación de organizaciones prestadoras de servicios de Bienestar social: Una aproximación conceptual. Revista Internacional Organizaciones [Internet]. 2006 [citado 4 Sept 2011]. Disponible en: http://www.revistario.org/index.php/revista_rio/article/view/7/7

9. Ministerio de Salud Pública. Programa Nacional para la Erradicación del mosquito Aedes aegypti. La Habana; 1982. Disponible en: http://www.ops.org. bo/textocompleto/iden28098.pdf

10. Ministerio de Salud Pública. Programa de Prevención del Dengue y Erradicación del Aedes Aegypti: Etapa de sostenibilidad. La Habana: MINSAP; 2007.

11. Campaña Anti-aegypti. Manual para responsables de brigadas y visitadores. La Habana: MINSAP; 1997.

12. González Gil E, Armada JA, Trigo JA. Metodología de encuestas. Campaña Antiaegypti. La Habana: MINSAP; 1997.

13. González Gil E, Armada JA, Trigo J A. Técnica de lucha anti-aegypti. La Habana: MI NSAP; 1997.

http://scielo.sld.cu 
14. La integración de la Higiene y la Epidemiología a la Atención Primaria de Salud. La Habana: MINSAP; 1995.

15 Dirección Nacional de Servicios Ambulatorios. Manual de organización del policlínico. La Habana: MINSAP; 2005.

16. Noriega V, Ponce Y, Pardo A y Pría MC. Ejercicio estratégico con expertos de Unidades de Vigilancia y Lucha Antivectorial de Ciudad Habana. Rev Infodir [Internet]. 2008 [citado 31 May 2010]; (7). Disponible en:

http://www.sld.cu/galerias/pdf/sitios/infodir/ejercicio_estrategico_con_expertos_de_u ats.pdf

17. Astigarraga E. El método Delphi. Universidad de Deusto San Sebastián [Internet]. 2005 [citado 20 nov 2011]. Disponible en:

http://www.prospectiva.eu/zaharra/03_Delphi_ESTE.pdf

18. Mallo PE, Artola MA, Galante MJ, Martínez D, Pascual ME, Morettini M. Aplicación del Método Delphi a las decisiones financieras en situaciones de incertidumbre[Internet]. Argentina: Universidad Nacional de Mar del Plata, Facultad de Ciencias Económicas y Sociales, 2012 [citado 2 Abr 2012]. Disponible en:

http://nulan.mdp.edu.ar/913/1/00179.pdf

19. Pineault R, Daveluy C. La planificación sanitaria. Conceptos, Métodos y Estrategias. Barcelona: Masson S.A; 1988.

20. Morejón Díaz Y. Evaluación de la efectividad del tratamiento focal contra el Aedes aegypti. Área de salud Párraga [tesis]. La Habana: Escuela Nacional de Salud Pública, 2007 [citado 20 nov 2011]. Disponible en:

http://www.sld.cu/galerias/pdf/sitios/revsalud/tesisyanet(7.1.09).pdf

21. Rojo Pérez N. Administración de recursos humanos. Generalidades en salud. En: Carnota O. Biblioteca Virtual para formación postgraduada de directivos del sector salud [CD-ROM]. La Habana: ENSAP; 2010.

22. Molina M. Estudios de clima organizacional [Internet]. 2008 [citado 14 Sept 2011]. Disponible en: http://www. losrecursoshumanos. com/contenidos /290estudios-de-clima-organizacional.html

23. Noriega Bravo V, Pría Barros MC. Instrumento para evaluar clima organizacional en los Grupos de Control de Vectores. Rev Cubana Salud Pública. [Internet]. 2011 [citado 17/5/2011];37(2), Disponible en:

http://bvs.sld.cu/revistas/spu/vol37_02_11/spu04211.htm

Recibido: 2 de mayo de 2012.

Aprobado: 31 de julio de 2012.

Vivian de las Mercedes Noriega Bravo. Escuela Nacional de Salud Pública. Calle 100 No. 1132 e/ E y Perla. Altahabana, Municipio Boyeros. La Habana, Cuba. Correo electrónico: vivy@infomed.sld.cu 